\title{
Emisión de compuestos tóxicos del aire de fuentes móviles en la Gran Área Metropolitana de Costa Rica en 2007
}

\section{Emissions of Air Toxic Compounds in Mobile Source for the Greater Metropolitan Area of Costa Rica in 2007}

\section{Javier E. Rodríguez-Yáñez ${ }^{1}$}

Fecha de recepción: 28 de marzo del 2014

Fecha de aprobación: 3 de febrero del 2015

Rodríguez-Yáñez, J. Emisión de compuestos tóxicos del aire de fuentes móviles en la Gran Área Metropolitana de Costa

Rica en 2007. Tecnología en Marcha. Vol. 28, № 4, Octubre-

Diciembre. Pág 66-77.

1 Ingeniero químico, uruguayo. Química Marina, Escuela de Química, Universidad Nacional. Campus Omar Dengo, Apdo. 863000, Heredia, Costa Rica. Teléfono: (506)2277-3351.

Correo electrónico: urutico@gmail.com 


\section{Palabras clave}

Fuentes móviles; tóxico; emisiones; GAM, Costa Rica.

\section{Resumen}

Se realizó una estimación de las emisiones de compuestos tóxicos generados por la flota vehicular en la Gran Área Metropolitana de Costa Rica para el año 2007. El cálculo se realizó mediante factores de emisión obtenidos con el modelo MOBILE 6 de la EPA, así como los datos de actividad aportados por la Dirección Sectorial de Energía y el Ministerio de Obras Públicas y Transportes.

Del total de 914.486 toneladas emitidas por fuentes móviles, de los contaminantes tóxicos evaluados, el $46 \%$ está asociada al benceno y el $26,8 \%$ al formaldehído. Las emisiones provienen principalmente de automóviles particulares y microbuses o transporte de carga liviana a gasolina, que representan el $70 \%$ del total.

\section{Keywords}

Mobile sources; toxic; emissions; GAM; Costa Rica.

\section{Abstract}

We estimated emissions of toxic compounds generated by the vehicle fleet in the GAM for 2007. This calculation was performed using emission factors obtained with the model of the EPA MOBILE 6 and activity data provided by the Energy Sector Management and the Ministry of Public Works and Transportation.

Of the total 914,486 tons emitted by mobile sources of toxic pollutants evaluated, we have that $46 \%$ is associated with Benzene and Formaldehyde $26.8 \%$. The emissions are mainly caused by private cars and light duty cargo or minibuses of gasoline powered, which represent $70 \%$ of total emissions.

\section{Introducción}

Los vehículos automotores que circulan por las carreteras son aquellos como automóviles, camiones y autobuses diseñados para operar en las vías públicas. En la mayor parte de las áreas urbanas, los vehículos automotores contribuyen en gran medida a las emisiones de gases al ambiente. Dada la gran magnitud de sus emisiones y las consideraciones especiales que se requieren para calcularlas, los vehículos automotores se tratan separadamente de otras fuentes de emisión (trenes, barcos o aviones).

Las emisiones vehiculares principales están constituidas por dióxido de carbono (CO2) compuestos volátiles orgánicos (VOC), monóxido de carbono (CO), óxido de nitrógeno (NOx), óxidos de azufre (SO2), partículas (PM). Algunas de estas especies reducen la visibilidad y otras son compuestos tóxicos del aire, en los cuales se enfoca este trabajo.

Dichas emisiones resultan de varios procesos diferentes que se presentan en la figura 1. Las emisiones más comúnmente consideradas son las del escape, que resultan de la combustión y se emiten por el tubo de escape del vehículo, y las que provienen de varios procesos de emisión evaporativa. 
Las metodologías de estimación de emisiones utilizan programas de modelado computacional, que permiten generar factores asociados a los diferentes tipos de vehículos y combustibles.

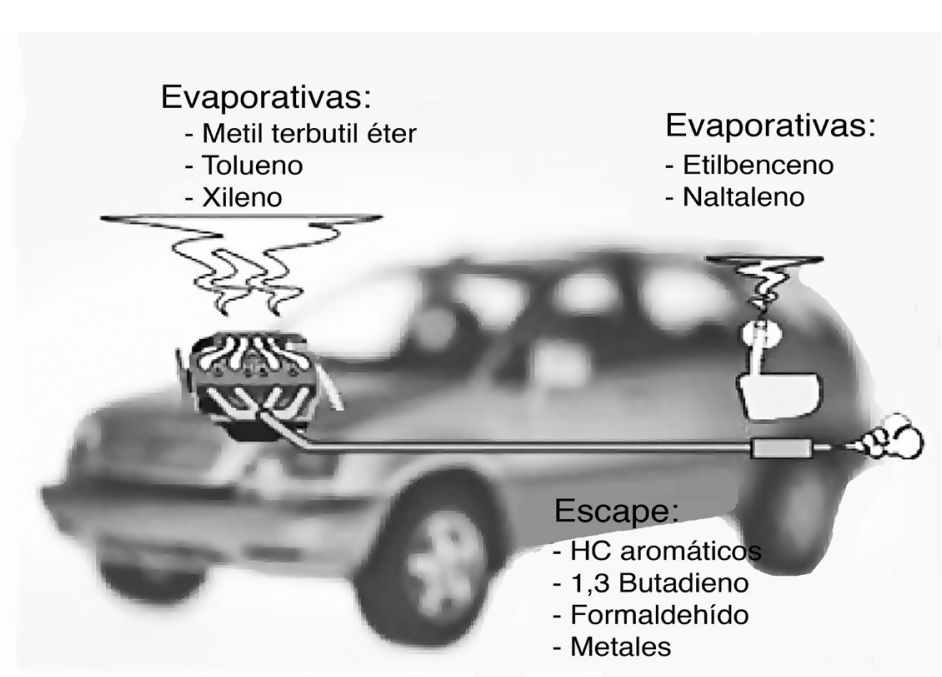

Figura 1. Tipos de emisiones y contaminantes tóxicos de fuentes móviles (ZMVM, 2004)

\section{Metodología}

La metodología utilizada para el estudio se basa en las consideraciones de aplicación del programa MOBILE 6 de la Agencia de Protección Ambiental de Estados Unidos (EPA), con el cual se obtienen factores de emisión considerando las características de la flota vehicular y las condiciones de escenario (clima, carreteras, etc.). A partir de estos factores es posible realizar el cálculo de estimación de emisiones considerando la actividad vehicular.

La ecuación básica utilizada para estimar las emisiones de los vehículos automotores involucra los datos de actividad vehicular y un factor de emisión.

$$
\mathrm{Ep}=\mathrm{KRV} \times \mathrm{FEp}
$$

donde:

$$
\begin{aligned}
& E_{p}=\text { Emisiones totales del contaminante } p \\
& K R V=\text { Kilómetros recorridos por el vehículo } \\
& F_{p}=\text { Factor de emisión del contaminante } p
\end{aligned}
$$

Para los vehículos automotores, los datos de actividad se refieren a los kilómetros recorridos por el vehículo (KRV), mientras que los factores de emisión se expresan en unidades de gramos de contaminante por KRV. Los KRV representan la distancia total recorrida por una población de vehículos en un periodo de tiempo determinado, siendo preferible estimar los KRV a partir de modelos de transporte o de conteos de vehículos en circulación. En algunos casos, sin embargo, los KRV deben calcularse a partir de las estadísticas de consumo de combustible (Wark y Warne, 2007).

Para el cálculo de los factores de estimación de emisiones (FEp) se utilizó el MOBILE 6 de la EPA, que define los principales compuestos tóxicos emitidos por fuentes móviles. 
El MOBILE 6 es un programa de cómputo integrado por rutinas elaboradas en lenguaje de programación Fortran y se utiliza para el cálculo de factores de emisión para distintos tipos de vehículos con motores a gasolina y diesel. El programa calcula factores de emisión para 28 tipos diferentes de categorías vehiculares, asociadas principalmente al tipo de vehículo, combustible, peso y tecnología de motor. Además, permite plantear la estimación para 25 años de los vehículos considerados dentro del periodo 1952-2050, en este caso entre 1983 y 2007 (Herrera, 2012).

Estos tóxicos se asocian a otros parámetros característicos de la flota vehicular, tales como la edad y el tipo de vehículo, según definiciones previas del programa, así como a consideraciones sobre el combustible, la circulación y tipos de carreteras. Otros factores considerados son las características climáticas del área y, dependiendo de la disponibilidad de información o aproximaciones, se pueden incluir otros parámetros, tales como material particulado, condiciones legales, etc. Con este conjunto de características se plantea un escenario para el área de estudio. En este artículo se presenta la estimación de las emisiones de compuestos tóxicos del aire en la Gran Área Metropolitana (GAM) de Costa Rica para el año 2007 (Rodríguez, 2012).

\section{Flota vehicular}

Se dividió la flota vehicular en ocho categorías de vehículos a partir de datos de los derechos de circulación del Instituto Nacional de Seguros (INS), sobre los que se hicieron las siguientes consideraciones:

Del total de la flota de vehículos en circulación, se consideró que el 57\% circulaba en la GAM, a partir de una estimación de venta de combustible en dicha área respecto al resto del país aportada por la Autoridad Reguladora de Servicios Públicos (ARESEP).

Se identificó, a través del INS, que de los autos modelo 2008, el 40\% había sido inscrito durante 2007.

El parque automotor tiene un rango de antigüedades de más de 30 años, pero el programa de estimación de factores de emisión MOBILE 6 solo acepta categorías de hasta 25 años, por lo que se restringen las antigüedades, agrupándose los vehículos anteriores a 1983 en este año. De la misma forma los vehículos empadronados como 2008 pero que circularon en 2007 se integran al conteo de vehículos de 2007. La cuantificación por tipo de vehículo y año se presenta en el cuadro 1.

Las unidades de clasificación corresponden a: automóviles, carga liviana, carga pesada, autobuses, taxis, microbuses, motocicletas y bicimotos. No se utilizan las segregaciones por peso en las categorías base.

Para los efectos del cálculo de los factores de emisión, se consideraron como parte de la categoría de carga pesada los remolques y los tracto-camiones, los cuales se contaron como unidades independientes.

Se asume que los vehículos que circulan en la GAM tienen actividad los 365 días del año, despreciando la existencia del programa de restricción vehicular existente en la ciudad de San José, debido a que su área de cobertura no es significativa con respecto al área de estudio.

Las características de la flota vehicular se discutirán conjuntamente con los resultados.

\section{Condiciones de escenario}

Los factores de emisión se obtuvieron del modelado computacional del programa MOBILE 6 de la EPA, ajustado a las condiciones definidas como el escenario a modelar, en este caso la GAM en Costa Rica en 2007, descritas en el cuadro 2. 
Tecnología en Marcha,

70 Vol. 28, N. ${ }^{\circ}$ 4, Octubre-Diciembre 2015

Cuadro 1. Flota vehicular considerada por tipo, modelo y combustible, según categoría de clasificación de MOBILE 6

\begin{tabular}{|c|c|c|c|c|c|c|c|c|c|}
\hline Año & Automóviles & Automóviles & $\begin{array}{c}\text { Carga } \\
\text { liviana y } \\
\text { microbuses }\end{array}$ & $\begin{array}{l}\text { Carga } \\
\text { liviana }\end{array}$ & $\begin{array}{c}\text { Carga } \\
\text { pesada }\end{array}$ & Taxis & Taxis & Autobuses & $\begin{array}{c}\text { Motocicletas } \\
\text { y bicimotos }\end{array}$ \\
\hline modelo & gasolina & diesel & gasolina & diesel & diesel & gasolina & diesel & diesel & gasolina \\
\hline Anteriores & 10865 & 21 & 771 & 5700 & 1455 & 0 & 0 & 2 & 915 \\
\hline 1983 & 1216 & 2 & 31 & 229 & 86 & 0 & 0 & 0 & 81 \\
\hline 1984 & 3121 & 7 & 103 & 762 & 290 & 0 & 0 & 1 & 87 \\
\hline 1985 & 3728 & 8 & 183 & 1352 & 503 & 0 & 0 & 0 & 165 \\
\hline 1986 & 5561 & 11 & 417 & 3086 & 402 & 0 & 0 & 3 & 161 \\
\hline 1987 & 11177 & 22 & 587 & 4344 & 574 & 1 & 0 & 2 & 250 \\
\hline 1988 & 13208 & 26 & 354 & 2614 & 633 & 1 & 0 & 6 & 256 \\
\hline 1989 & 10606 & 22 & 393 & 2908 & 700 & 0 & 0 & 22 & 359 \\
\hline 1990 & 11261 & 22 & 421 & 3115 & 717 & 0 & 0 & 100 & 505 \\
\hline 1991 & 17258 & 35 & 332 & 2453 & 539 & 2 & 0 & 136 & 504 \\
\hline 1992 & 22186 & 44 & 265 & 1951 & 597 & 2 & 0 & 182 & 589 \\
\hline 1993 & 22020 & 44 & 343 & 2536 & 860 & 6 & 1 & 249 & 829 \\
\hline 1994 & 22114 & 44 & 352 & 2605 & 847 & 793 & 70 & 392 & 1242 \\
\hline 1995 & 19075 & 38 & 331 & 2446 & 1220 & 693 & 62 & 495 & 923 \\
\hline 1996 & 15255 & 31 & 191 & 1410 & 975 & 414 & 37 & 421 & 880 \\
\hline 1997 & 14592 & 30 & 212 & 1549 & 836 & 411 & 37 & 400 & 706 \\
\hline 1998 & 11502 & 23 & 288 & 2127 & 1024 & 193 & 17 & 354 & 936 \\
\hline 1999 & 12029 & 24 & 323 & 2377 & 1060 & 251 & 22 & 466 & 536 \\
\hline 2000 & 12079 & 24 & 301 & 2203 & 1086 & 492 & 44 & 516 & 599 \\
\hline 2001 & 11663 & 23 & 235 & 1716 & 520 & 398 & 35 & 495 & 658 \\
\hline 2002 & 10617 & 21 & 246 & 1811 & 274 & 358 & 32 & 464 & 1035 \\
\hline 2003 & 9093 & 18 & 290 & 2142 & 492 & 162 & 14 & 518 & 1222 \\
\hline 2004 & 7499 & 15 & 216 & 1585 & 206 & 191 & 17 & 337 & 1551 \\
\hline 2005 & 8031 & 16 & 234 & 1720 & 147 & 333 & 30 & 439 & 2729 \\
\hline 2006 & 8845 & 18 & 346 & 2481 & 135 & 384 & 34 & 355 & 7399 \\
\hline 2007 & 10583 & 21 & 459 & 3150 & 240 & 295 & 26 & 505 & 11823 \\
\hline 2008 & 5983 & 12 & 282 & 2002 & 189 & 117 & 10 & 211 & 7968 \\
\hline Total & 311.167 & 622 & 8.506 & 62.374 & 16.607 & 5.497 & 488 & 7.071 & 44.908 \\
\hline$\%$ & $68,05 \%$ & $0,14 \%$ & $1,86 \%$ & $13,64 \%$ & $3,63 \%$ & $1,20 \%$ & $0,11 \%$ & $1,55 \%$ & $9,82 \%$ \\
\hline
\end{tabular}


Cuadro 2. Condiciones de la GAM para la aplicación del programa MOBILE 6

\begin{tabular}{|c|c|}
\hline Parámetro MOBILE 6 & Datos de entrada proporcionados \\
\hline Región & $\begin{array}{c}\text { Ciudad a una altitud menor o igual a } 5500 \\
\text { pies }(1677 \mathrm{~m})\end{array}$ \\
\hline Año calendario por evaluar & 2007 \\
\hline Velocidad promedio de circulación en la región & $33,1 \mathrm{~km} / \mathrm{h}^{(1)}$ \\
\hline Temperatura máxima diaria para la región & $25,8^{\circ} \mathrm{C}^{(2)}$ \\
\hline Temperatura mínima diaria para la región & $17,4^{\circ} \mathrm{C}^{(2)}$ \\
\hline Presión de Vapor Reid de la gasolina & $63,5 \mathrm{kPa}^{(3)}$ \\
\hline Contenido de azufre en diesel & 2500 ppm (3) \\
\hline Contenido de azufre en gasolina & 192,5 ppm (3) \\
\hline Contenido de oxigenados en gasolina & Menor a $1 \%{ }^{(3)}$ \\
\hline Porcentaje de vapor en la gasolina a $200^{\circ} \mathrm{F}$ & $50^{(3)}$ \\
\hline Porcentaje de vapor en la gasolina a $300^{\circ} \mathrm{F}$ & $85^{(3)}$ \\
\hline Porcentaje de aromáticos en la gasolina & $23^{(3)}$ \\
\hline Porcentaje de olefinas en la gasolina & $20^{(3)}$ \\
\hline Porcentaje de benceno en la gasolina & $0,8^{(3)}$ \\
\hline
\end{tabular}

(1) PRUGAM, Transporte, 2009.

(2) Instituto Meteorológico Nacional.

(3) ARESEP, 2011.

En el MOBILE 6 utilizado, los factores de emisión de tóxicos se definen considerando sus emisiones en forma evaporativa y de escape como fracción de emisión en $\mathrm{g} / \mathrm{milla}$ recorrida (1 milla=1,609 km). Se considera que las emisiones evaporativas son solamente en vehículos a gasolina.

El MOBILE 6 tiene predefinidos los seis principales compuestos tóxicos más habituales: benceno, metil terbutil éter (MTEB), 1,3 butadieno, formaldehído, acetaldehído y acroleína. No se consideraron otros tóxicos.

Las fracciones de emisión de contaminantes tóxicos obtenidas a partir del MOBILE 6 se relacionan con las emisiones totales de tóxicos específicos a través de los kilómetros recorridos por los tipos y modelos.

Como parámetros del programa se consideraron los tipos de carretera (FTYPE) según lo estimado por el Ministerio de Obras Públicas y Transporte (MOPT) para el Primer Inventario de Contaminantes Criterio del GAM en 2007 (Herrera, 2011) y los tipos de emisión asociados a los vehículos (ETYPE) especificados en el MOBILE.

\section{Actividad vehicular}

Los valores de actividad expresados como km recorrido por tipo de vehículo se obtuvieron a través de metodologías aplicadas en la GAM, desarrolladas por la Dirección Sectorial de Energía (DSE) a partir de encuestas, y se expresan en el cuadro 3 (DSE, 2009). 
Cuadro 3. Estimación de la circulación según la DSE

\begin{tabular}{|c|c|c|}
\hline Tipo de vehículo & km/día & km/año \\
\hline Automóviles particulares & 57 & 20768 \\
\hline Taxis & 230 & 86109 \\
\hline Carga liviana & 77 & 27990 \\
\hline Carga pesada & 107 & 38934 \\
\hline Motocicletas & 43 & 16091 \\
\hline Autobuses & 118 & 4417 \\
\hline
\end{tabular}

Con los datos del recorrido diario, los días que circularon los vehículos durante el año 2007 y el número de vehículos de acuerdo con la distribución por año modelo, se obtuvieron los kilómetros recorridos (KRV) por tipo de vehículo y año modelo, a partir de la ecuación:

donde:

$$
\mathrm{KRV}_{\mathrm{ij}}=\left(\mathrm{KD}_{\mathrm{j}}\right)\left(\mathrm{NV}_{\mathrm{ij}}\right)\left(\mathrm{DA}_{\mathrm{i}}\right)
$$

$\mathrm{KRV}_{\mathrm{ij}}=$ Kilómetros recorridos por el tipo de vehículo i, del año modelo j [km/año].

$\mathrm{KD}_{\mathrm{j}}=$ Kilómetros recorridos al día por el tipo de vehículo i [km/día].

$\mathrm{NV}_{\mathrm{ij}}=$ Número de vehículos del tipo i, del año modelo j.

$\mathrm{DA}_{\mathrm{i}}=$ Días al año que circulan los vehículos del tipo i [días/año].

\section{Cálculo de emisión}

A partir de lo anterior, es posible obtener la estimación de los valores de emisión anuales para los compuestos tóxicos del aire en la GAM, de acuerdo con una multiplicación relativa según:

$$
E_{i j k}=\left(k E I M_{i j}\right)\left(F E T_{i j k}\right) /(1,609 * 1.000 .000)
$$

donde:

$\mathrm{E}_{\mathrm{ijk}}=$ Emisión del tipo de vehículo i, año modelo j, del contaminante $\mathrm{k}$ [t/año].

$\mathrm{kEIM}_{\mathrm{ij}}=\mathrm{km}$ recorridos según EIM por categoría de clasificación del tipo de vehículo i, año modelo j [km/año].

$\mathrm{FET}_{\mathrm{ijk}}=$ Factor de emisión de tóxicos como g/milla por categoría de clasificación del tipo de vehículo i, año modelo j, del contaminante $\mathrm{k}$ [g/milla]

1.000.000 = Factor de conversión de gramos a toneladas.

1,609 = Factor de conversión de millas a km.

Se calculan así las emisiones de cada contaminante para cada tipo de vehículo, modelo y año. Luego dichas emisiones se suman para obtener la emisión global de cada contaminante por fuentes móviles.

\section{Resultados y discusión}

La estimación de emisiones se resume en el cuadro 4, en el que se indican las emisiones totales de contaminantes tóxicos del aire por tipo de emisión. 
Cuadro 4. Emisiones totales en t/año por tipo de emisión y contaminante

\begin{tabular}{|c|c|c|c|c|}
\hline Tóxico & Escape (t/año) & $\begin{array}{c}\text { Evaporación } \\
\text { (t/año) }\end{array}$ & Total (t/año) & $\begin{array}{c}\text { \% de fuentes } \\
\text { móviles }\end{array}$ \\
\hline Benceno & 421,217 & & 421,22 & $46,06 \%$ \\
\hline Formaldehído & 245,188 & & 245,19 & $26,81 \%$ \\
\hline 1,3 Butadieno & 114,377 & & 114,38 & $12,51 \%$ \\
\hline Acetaldehído & 90,599 & & 90,60 & $9,91 \%$ \\
\hline MTBE & 7,340 & 24,146 & 31,49 & $3,44 \%$ \\
\hline Acroleína & 11,620 & & 11,62 & $1,27 \%$ \\
\hline TOTAL & 890,340 & 24,146 & 914,486 & \\
\hline
\end{tabular}

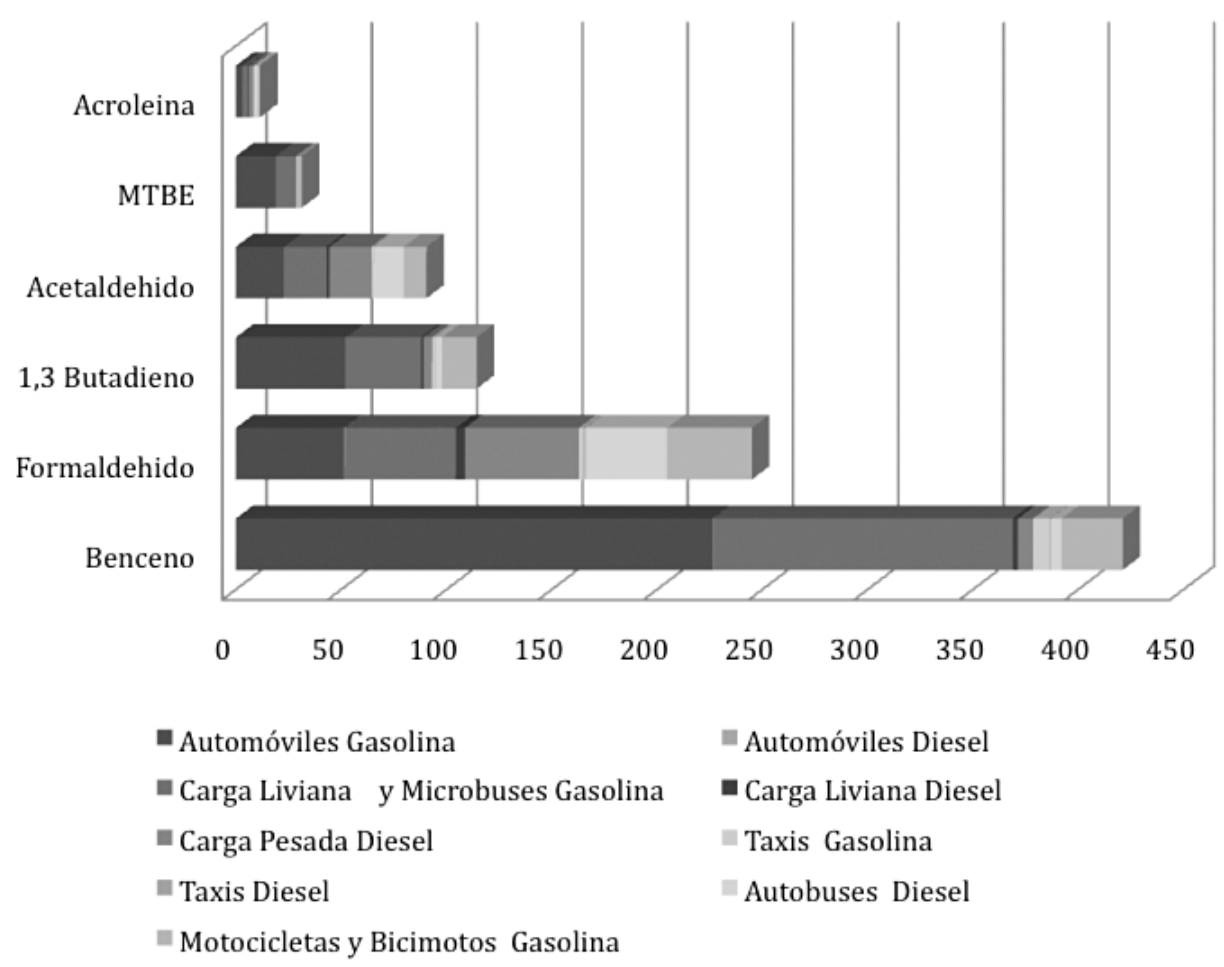

Figura 2. Emisiones totales por contaminante en t/año por tipo de vehículo

Las emisiones totales estimadas asociadas a fuentes móviles son aproximadamente 914,486 t/ año para la GAM en 2007.

Del cuadro 4 se desprende que las emisiones principales se asocian con escapes, teniendo como los contaminantes más importantes el benceno y el formaldehído, seguidos en segundo orden por 1,3 butadieno, acetaldehído, MTBE y acroleína.

En el cuadro 5 y la figura 2 se aprecia que la categoría de Automóviles particulares a gasolina es la que presenta el mayor aporte en casi todos los contaminantes tóxicos considerados, pero afectando especialmente a la emisión de benceno. En segundo término los aportes de Microbuses y Carga liviana a gasolina, así como de Motocicletas y Bicimotos son los más 
importantes, en ese orden. Las categorías Carga pesada y Autobuses a diesel también afectan en forma importante la emisión de formaldehído.

La distribución por edad muestra que hay un 23,3\% de vehículos anteriores a 1991, los cuales no tienen tecnologías de control de emisiones. De estos, un 4,3\% es anterior a 1983. El periodo 1991 y 1992 plantea una etapa de transición a vehículos con nuevas tecnologías de control de emisiones, representando un 10,3\%.

La cantidad de automóviles a gasolina aumentó mucho a inicios de los años noventa, pasando de unos 5000 vehículos al año a mediados de los ochenta a más de 20.000, estabilizándose para el año 2000 en unos 10.000 vehículos por año. A partir del año 2000 se inicial el aumento de otros vehículos alternativos, en especial microbuses y motocicletas.

En general, para todos los tóxicos considerados, los vehículos más viejos son los que emiten en mayor proporción los contaminantes, debido a la falta de tecnologías de control, representando en promedio 50-60\% de las emisiones en cada contaminante, como puede verse en la figura 3.

Como situaciones particulares, se tiene que para las Motocicletas y Bicimotos el $60 \%$ de la emisión está asociada a vehículos posteriores a 1992, mientras que para Autobuses, dadas las limitaciones de edad de las flotas, el 90\% de las emisiones está asociado a modelos posteriores a 1992.

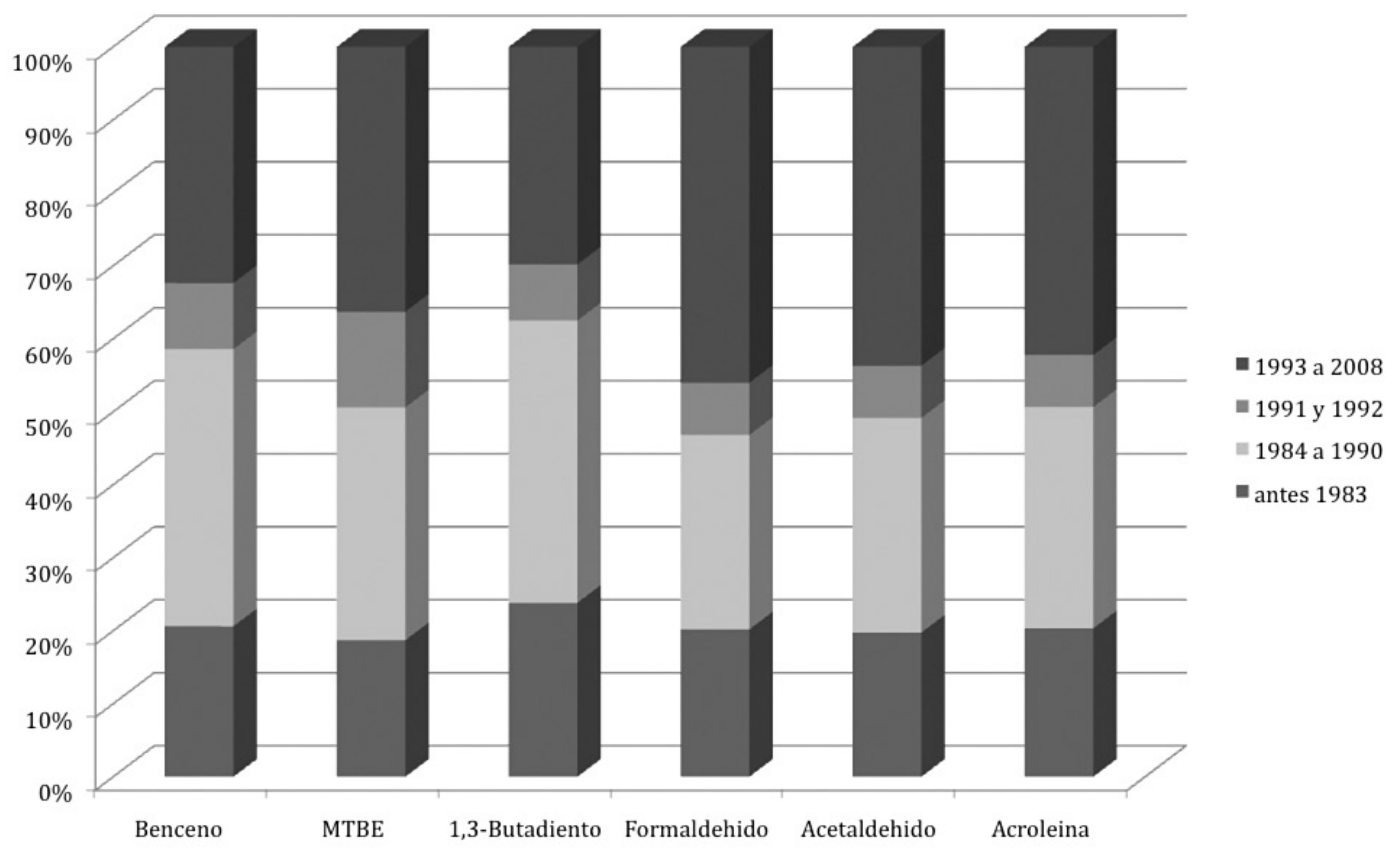

Figura 3. Distribución de misiones por edad de los vehículos 


\begin{tabular}{|c|c|c|c|c|c|c|c|c|c|}
\hline 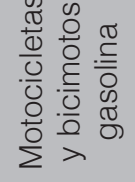 & 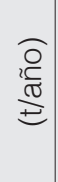 & $\begin{array}{l}\text { Na } \\
\text { o, } \\
\text { o } \\
\text { N }\end{array}$ & $\begin{array}{l}\text { ᄋ } \\
\text { m } \\
\text { o }\end{array}$ & 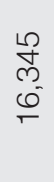 & $\begin{array}{l}5 \\
0 \\
0 \\
0\end{array}$ & $\frac{m}{\sigma}$ & 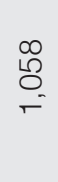 & $\begin{array}{l}\text { Oे } \\
\text { ले } \\
\text { ठ } \\
\text { ○ }\end{array}$ & \begin{tabular}{l}
$\stackrel{0}{\circ}$ \\
$\hat{\circ}$ \\
$\circ$ \\
\multicolumn{1}{c}{}
\end{tabular} \\
\hline $\begin{array}{l}\infty \\
0 \\
0 \\
\frac{0}{1} \\
\frac{0}{0} \\
\frac{0}{0} \\
\frac{1}{2}\end{array}$ & 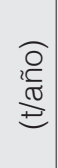 & 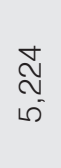 & $\begin{array}{l}\stackrel{1}{ } \\
8 \\
\infty \\
\infty \\
\infty\end{array}$ & 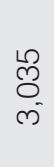 & $\begin{array}{l}\infty \\
\stackrel{N}{N} \\
\underset{\leftarrow}{\leftarrow}\end{array}$ & 0 & 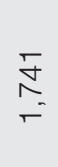 & $\begin{array}{l}\text { } \\
\stackrel{N}{ } \\
\tilde{ల}^{-}\end{array}$ & $\begin{array}{l}\circ \\
\frac{0}{0} \\
0\end{array}$ \\
\hline $\begin{array}{ll}\stackrel{\infty}{\Phi} & \bar{\Phi} \\
\stackrel{\varpi}{\varpi} & \stackrel{\infty}{\overline{0}}\end{array}$ & $\begin{array}{l}\overparen{O} \\
2 \\
\mathbb{0} \\
\pm \\
\pm\end{array}$ & $\begin{array}{l}\mathcal{N} \\
\text { N } \\
0 \\
0\end{array}$ & $\begin{array}{l}8 \\
8 \\
0 \\
0 \\
0\end{array}$ & 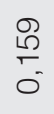 & 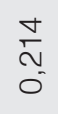 & 0 & $\begin{array}{l}\widetilde{0} \\
8 \\
0 \\
0^{-}\end{array}$ & $\begin{array}{l}\hat{\theta} \\
\dot{q} \\
r\end{array}$ & $\begin{array}{l}\stackrel{0}{0} \\
\stackrel{0}{\circ}\end{array}$ \\
\hline 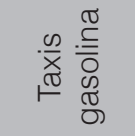 & 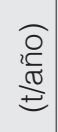 & $\begin{array}{l}\bar{\sigma} \\
\infty \\
\stackrel{2}{N} \\
\end{array}$ & $\begin{array}{l}\infty \\
\infty \\
\stackrel{0}{N}\end{array}$ & 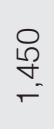 & $\begin{array}{l}0 \\
10 \\
\infty \\
0 \\
0\end{array}$ & $\begin{array}{l}\widetilde{\gamma} \\
8 \\
0 \\
0\end{array}$ & $\frac{10}{5}$ & $\begin{array}{l}0 \\
\stackrel{0}{0} \\
\stackrel{1}{T}\end{array}$ & $\begin{array}{l}\stackrel{0}{\circ} \\
\hat{\rho} \\
\leftarrow\end{array}$ \\
\hline 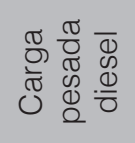 & 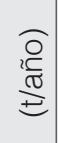 & 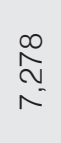 & 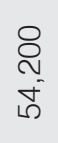 & $\begin{array}{l}\stackrel{\infty}{\sim} \\
\underset{\forall}{*}\end{array}$ & $\begin{array}{l}\overline{6} \\
8 \\
0 \\
\end{array}$ & 0 & $\begin{array}{l}\stackrel{0}{\sim} \\
\stackrel{v}{N}\end{array}$ & $\begin{array}{l}\text { Oे } \\
0^{-} \\
\infty \\
\infty\end{array}$ & $\begin{array}{l}\circ \\
\text { ஸे } \\
6 \\
\sigma^{\circ}\end{array}$ \\
\hline 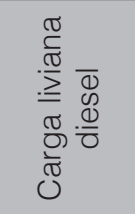 & 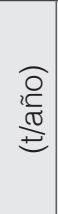 & $\begin{array}{l}\bar{L} \\
\stackrel{i n}{N}\end{array}$ & $\begin{array}{l}\stackrel{m}{\text { p}} \\
\stackrel{+}{\sim}\end{array}$ & $\stackrel{m}{\infty}$ & 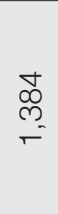 & 0 & $\begin{array}{l}\text { J } \\
\text { లె } \\
0\end{array}$ & $\begin{array}{l}\infty \\
\infty \\
0 \\
\sigma^{\infty}\end{array}$ & $\stackrel{\circ}{\stackrel{一}{\circ}}$ \\
\hline 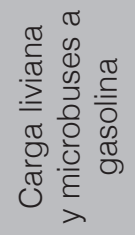 & 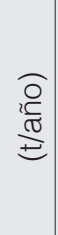 & $\begin{array}{l}\bar{m} \\
\stackrel{5}{v} \\
\stackrel{f}{\sim}\end{array}$ & 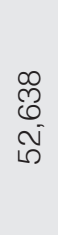 & $\begin{array}{l}m \\
\infty \\
\llcorner \\
10 \\
\infty\end{array}$ & $\begin{array}{l}\nabla \\
\sigma \\
\sigma \\
\sigma \\
\sigma\end{array}$ & $\begin{array}{l}8 \\
\dot{0} \\
\dot{\sigma}\end{array}$ & $\begin{array}{l}0 \\
0 \\
i\end{array}$ & $\begin{array}{l}\hat{\infty} \\
\hat{\alpha} \\
\text { ô } \\
\text { N }\end{array}$ & $\begin{array}{l}\stackrel{\circ}{\stackrel{\circ}{+}} \\
\stackrel{\infty}{\sim}\end{array}$ \\
\hline 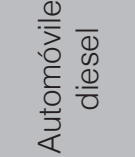 & 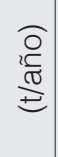 & $\begin{array}{l}\hat{m} \\
\overline{0} \\
\overline{0}\end{array}$ & 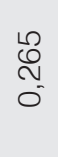 & $\begin{array}{l}\tilde{O} \\
\mathscr{8} \\
0-\end{array}$ & $\begin{array}{l}+ \\
0 \\
0 \\
0 \\
0\end{array}$ & 0 & $\begin{array}{l}\stackrel{+}{N} \\
\text { O- } \\
\text { O- }\end{array}$ & $\begin{array}{l}\widetilde{N} \\
\hat{n} \\
0\end{array}$ & $\begin{array}{l}\stackrel{\circ}{8} \\
\stackrel{0}{0}\end{array}$ \\
\hline 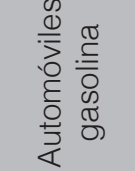 & 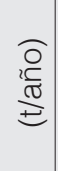 & 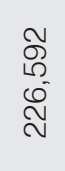 & 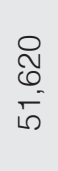 & 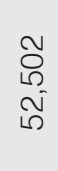 & $\frac{\stackrel{N}{N}}{\stackrel{N}{N}}$ & $\begin{array}{l}10 \\
8 \\
0 \\
\infty \\
\infty\end{array}$ & 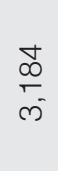 & $\begin{array}{l}\hat{m} \\
0 \\
0 \\
\hat{m}\end{array}$ & 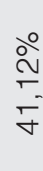 \\
\hline $\begin{array}{l}\frac{0}{7} \\
\frac{0}{2} \\
\frac{0}{10} \\
0 \\
0 \\
0 \\
0 \\
\stackrel{0}{=}\end{array}$ & 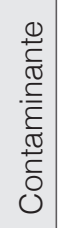 & 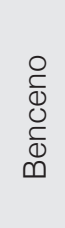 & $\begin{array}{l}\frac{0}{\frac{0}{2}} \\
\frac{0}{0} \\
\frac{0}{0} \\
\frac{0}{0} \\
\frac{0}{0} \\
\frac{1}{4}\end{array}$ & $\begin{array}{l}0 \\
\frac{0}{0} \\
\frac{0}{0} \\
\frac{\pi}{J} \\
5 \\
0 \\
m \\
m \\
-\end{array}$ & $\begin{array}{l}\frac{0}{0} \\
\frac{0}{0} \\
\frac{0}{0} \\
\frac{0}{00} \\
\frac{10}{0} \\
\frac{8}{4}\end{array}$ & 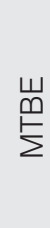 & 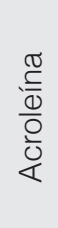 & 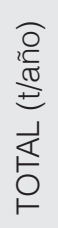 & $\circ^{\circ}$ \\
\hline
\end{tabular}




\section{Conclusiones}

En la GAM, las fuentes móviles generan un total de 914,486 toneladas de contaminantes tóxicos del aire. Dentro de los contaminantes evaluados, el principal es benceno, con un $46 \%$, seguido del formaldehído, con un 26,8\%. Los vehículos a gasolina aportan principalmente benceno, mientras que los de diesel lo hacen con formaldehído.

Los vehículos que más contaminantes producen son los Automóviles particulares, seguidos por los Microbuses o de Carga liviana a gasolina, que aportan en conjunto el $70 \%$ de las emisiones totales. Los primeros por su gran proporción en el parque vehicular (68\%) y circulación, mientras que los segundos por el nivel de emisión (31 g/vehículo). Las Motocicletas son el tercer tipo de vehículos a gasolina con un porcentaje cercano al 11\% de la emisión global.

Paralelamente, los vehículos de Carga pesada y Autobuses, que representan solo el 5,2\% de la flota vehicular, emiten el 16,5\% del total de emisiones.

Resulta de especial interés que los vehículos sin tecnologías de control de emisiones, anteriores a 1991, que son el 23,3\% de la flota vehicular, generan el 54,6 \% de las emisiones globales.

Estas relaciones son similares en otros inventarios para los Automóviles particulares, Motocicletas y Autobuses, en los que se marca de forma importante la utilización del transporte vehicular en la GAM.

Se destaca en este caso una diferencia importante en cuanto a las categorías asociadas al transporte de carga en la GAM, dado el nivel de emisión obtenido con un bajo porcentaje de vehículos. Esto podría explicarse por la actividad comercial e industrial en dicha área y las dificultades de circulación de cargas, convirtiéndose en un cruce de la red vial del país sin muchas alternativas de circulación rápida o fuera de ella.

\section{Agradecimientos}

Se agradece el apoyo del Laboratorio de Análisis Ambiental de la Universidad Nacional y la Dirección de Gestión de Calidad Ambiental, así como del Ministerio de Ciencia y Tecnología, del cual el Ing. Javier Rodríguez es becario.

\section{Bibliografía}

Dirección Sectorial de Energía. (2009). Encuesta del recorrido medio anual de los vehículos en circulación en Costa Rica 2009. Recuperado de www.dse.go.cr/es/03Publicaciones/04Tecnicas/RecorridoVehículosCR2009.pdf

Environmental Protection Agency (EPA). (2011). MOBILE 6 Vehicle Emission Modeling Software. Recuperado de www. epa.gov/oms/m6.htm

Finlayson-Pitts, B.J. \& Pitts J.N.Jr. (2004). Chemistry of the Upper and Lower Atmosphere. Academic Press.

Herrera J., Rojas, F., Rodríguez, S., Rojas, A. \& Rodríguez, J. (2011). Primer Inventario de Emisiones de Contaminantes Criterio del Aire para el Gran Área Metropolitana de Costa Rica en el 2007, Costa Rica. Recuperado de www. digeca.go.cr/documentos/aire/Resumen\%20ejecutivo\%20del\%20Inventario.pdf

Herrera, J., Rodríguez, S. \& Rojas J. (2012). Determinación de las emisiones de contaminantes del aire generadas por fuentes móviles en carreteras de Costa Rica. Tecnología en Marcha, 25(1), 54-63.

Instituto Nacional de Ecología. (2007). Manual para la elaboración y usos de inventarios de emisiones. México: Secretaría de Medio Ambiente.

MINAET, MOPT, MS, UNA. (2008). Plan Nacional para Mejorar la Calidad del Aire en el Gran Área Metropolitana de Costa Rica, 2008-2013.

PRUGAM. (2009). Plan Regional Urbano para el Gran Área Metropolitana de Costa Rica, 2008-2030, Costa Rica. Tomo VI Resumen Ejecutivo. 
Rodríguez J. (2012). Inventario de Compuestos Tóxicos Peligrosos del Aire para el GAM en 2007. Tesis de Maestría en Gestión y Estudios Ambientales, Universidad Nacional, Heredia, Costa Rica.

Secretaría de Medio Ambiente y Gobierno del Distrito Federal. (2006). Inventario de Emisiones de Contaminantes Tóxicos del Aire de la Zona Metropolitana del Valle de México en 2004 (ZMVM; 2004), México.

Secretaría de Medio Ambiente y Gobierno del Distrito Federal. (2010). Inventario de Emisiones de Contaminantes Tóxicos del Aire de la Zona Metropolitana del Valle de México en 2008 (ZMVM; 2008), México.

Subsecretaría de Gestión para la Protección Ambiental-SEMARNAT y Comisión para la Cooperación Ambiental de América del Norte. (2001). Guía para la Correcta Selección y Empleo de Métodos de Estimación de Emisiones Contaminantes, México.

Solano, J., \& Villalobos, R. (2000). Regiones y subregiones climáticas de Costa Rica. San José: Instituto Meteorológico Nacional. Instituto Meteorológico Nacional. Recuperado de www.imn.ac.cr

Wark, K. \& Warner, C.F. (2007). Contaminación del Aire, origen y control. México: Limusa. 